\title{
Evolution of bivariate copulas in discrete processes
}

\author{
Yasukazu Yoshizawa ${ }^{1}$ and Naoyuki Ishimura ${ }^{1}$ \\ ${ }^{1}$ Graduate School of Economics, Hitotsubashi University, 2-1 Naka Kunitachi, Tokyo 186-8601, \\ Japan \\ E-mailed091006@g.hit-u.ac.jp, ishimura@econ.hit-u.ac.jp
}

Received October 13, 2011, Accepted October 19, 2011

\begin{abstract}
A copula function makes a bridge between multivariate joint distributions and univariate marginal distributions, and provides a flexible way of describing nonlinear dependence among random circumstances. We introduce a new family of bivariate copulas which evolves according to the discrete process of heat equation. We prove the convergence of solutions as well as the measure of dependence. Numerical experiments are also performed, which shows that our procedure works substantially well.
\end{abstract}

Keywords copula, discrete processes, risk management

Research Activity Group Mathematical Finance

\section{Introduction}

There has been much interest in the theory of copulas these days. A copula technique provides a flexible and convenient method of describing nonlinear dependence among multivariate random events. Copulas make a link between a multivariate joint distribution and univariate marginal distributions. The technique is employed not only in statistics but also in many areas of applications, which include financial engineering, risk management, actuarial science, seismology and so on. We refer to [110] and the references therein.

In the case of bivariate joint distribution, the definition of copula and the fundamental theorem developed by A. Sklar [11] is expressed as follows.

Definition 1 A function $C$ defined on $I^{2}:=[0,1] \times$ $[0,1]$ and valued in $I$ is called a copula if the following conditions are fulfilled.

(i) For every $(u, v) \in I^{2}$,

$$
\begin{aligned}
& C(u, 0)=C(0, v)=0, \\
& C(u, 1)=u \quad \text { and } \quad C(1, v)=v .
\end{aligned}
$$

(ii) For every $\left(u_{i}, v_{i}\right) \in I^{2}(i=1,2)$ with $u_{1} \leq u_{2}$ and $v_{1} \leq v_{2}$,

$$
C\left(u_{1}, v_{1}\right)-C\left(u_{1}, v_{2}\right)-C\left(u_{2}, v_{1}\right)+C\left(u_{2}, v_{2}\right) \geq 0 .
$$

The requirement (2) is referred to as the 2-increasing condition. We also note that a copula is continuous by its definition.

Theorem 2 (Sklar's theorem) Let $H$ be a bivariate joint distribution function with marginal distribution functions $F$ and $G$; that is,

$$
\lim _{x \rightarrow \infty} H(x, y)=G(y), \quad \lim _{y \rightarrow \infty} H(x, y)=F(x) .
$$

Then there exists a copula, which is uniquely determined on $\operatorname{RanF} \times$ RanG, such that

$$
H(x, y)=C(F(x), G(y)) .
$$

Conversely, if $C$ is a copula and $F$ and $G$ are distribution functions, then the function $H$ defined by (3) is a bivariate joint distribution function with margins $F$ and $G$.

In this article, we introduce a new family of bivariate copulas, which evolves according to discrete process.

Although there exist many one-parameter families of copulas, such as the Clayton family, the GumbelHougaard family and the Frank family, little attention seems to have been paid to the time-dependent copulas despite its importance. We just recall one important exception of the concept of dynamic copula due to A. J. Patton [12].

On the other hand, we have introduced the time evolution of copulas in [13-15]. To be precise, we consider a time parameterized family of copulas $\{C(u, v, t)\}_{t \geq 0}$, which satisfy the heat equation:

$$
\frac{\partial C}{\partial t}(u, v, t)=\left(\frac{\partial^{2}}{\partial u^{2}}+\frac{\partial^{2}}{\partial v^{2}}\right) C(u, v, t) .
$$

Here, by the definition of copula, we understand that $C(\cdot, \cdot, t)$ fulfills $(1),(2)$; to be precisely, we postulate that

(i) for every $(u, v, t) \in I^{2} \times(0, \infty)$,

$$
\begin{aligned}
& C(u, 0, t)=C(0, v, t)=0, \\
& C(u, 1, t)=u \quad \text { and } \quad C(1, v, t)=v .
\end{aligned}
$$

(ii) for every $\left(u_{i}, v_{i}, t\right) \in I^{2} \times(0, \infty)(i=1,2)$ with $u_{1} \leq u_{2}$ and $v_{1} \leq v_{2}$,

$$
\begin{aligned}
& C\left(u_{1}, v_{1}, t\right)-C\left(u_{1}, v_{2}, t\right) \\
& \quad-C\left(u_{2}, v_{1}, t\right)+C\left(u_{2}, v_{2}, t\right) \geq 0 .
\end{aligned}
$$

The stationary solution to (4), which is referred to as the harmonic copula, is uniquely determined to be 
$\Pi(u, v):=u v$, in view of the boundary condition (1). We note that the copula $\Pi$ represents the independent structure between two respective random variables.

Here we discretize (4) in a sense, and define a timedependent family of copulas in discrete processes. We hope that these discretized families are rather ready to be numerically computed and to be applied in many situations. We exhibit some examples in Section 4.

\section{Discrete processes of copulas}

The construction of our discretely parametrized family of copulas proceeds as follows.

Let $N \gg 1$ and $0<h \ll 1$. We put

$$
\begin{aligned}
& \Delta u=\Delta v:=\frac{1}{N}, \quad \Delta t:=h, \\
& \lambda:=\frac{\Delta t}{(\Delta u)^{2}}=\frac{\Delta t}{(\Delta v)^{2}}=h N^{2},
\end{aligned}
$$

and

$$
\begin{aligned}
& u_{i}:=i \Delta u=\frac{i}{N} \quad \text { for } i=0,1, \ldots, N \\
& v_{j}:=j \Delta v=\frac{j}{N} \quad \text { for } j=0,1, \ldots, N .
\end{aligned}
$$

Our family of copulas $\left\{C^{n}(u, v)\right\}_{n=0,1,2, \ldots}$ is now defined as follows: First,

$$
C^{0}(u, v):=C_{0}(u, v)
$$

where $C_{0}$ denotes given initial copula.

At $\left\{\left(u_{i}, v_{j}\right)\right\}_{i, j=0,1, \ldots, N}$, the value $C_{i, j}^{n}:=C^{n}\left(u_{i}, v_{j}\right)$ is governed by the system of difference equations

$$
\begin{aligned}
& \frac{C_{i, j}^{n+1}-C_{i, j}^{n}}{\Delta t}= \frac{C_{i+1, j}^{n}-2 C_{i, j}^{n}+C_{i-1, j}^{n}}{(\Delta u)^{2}} \\
&+\frac{C_{i, j+1}^{n}-2 C_{i, j}^{n}+C_{i, j-1}^{n}}{(\Delta v)^{2}} \\
& \quad \text { for } i, j=1,2, \ldots, N-1,
\end{aligned}
$$

together with the boundary conditions

$$
\left\{\begin{array}{l}
C_{0, j}^{n}=0=C_{i, 0}^{n} \\
C_{i, N}^{n}=u_{i}, \quad C_{N, j}^{n}=v_{j}
\end{array} \quad \text { for } i, j=0,1, \ldots, N .\right.
$$

As to the point $(u, v) \in \boldsymbol{I}^{2}$ other than $\left\{\left(u_{i}\right.\right.$, $\left.\left.v_{j}\right)\right\}_{i, j=0,1, \ldots, N}$, the value $C^{n}(u, v)$ is provided by linear interpolation. That is, if for instance

$$
u_{i} \leq u \leq u_{i+1}, \quad v_{j} \leq v \leq v_{j+1}, \quad v-v_{j} \leq u-u_{i},
$$

then

$$
\begin{aligned}
C^{n}(u, v):= & C_{i, j}^{n}+\frac{C_{i+1, j}^{n}-C_{i, j}^{n}}{u_{i+1}-u_{i}}\left(u-u_{i}\right) \\
& +\frac{C_{i+1, j+1}^{n}-C_{i+1, j}^{n}}{v_{i+1}-v_{i}}\left(v-v_{j}\right) .
\end{aligned}
$$

Other parts are computed similarly.

It is easy to check that a sequence of copulas $\left\{C^{n}(u, v)\right\}_{n=0,1,2, \ldots}$ defined above verify the boundary conditions (1) as well as the 2-increasing condition (2) provided $\lambda \leq 1 / 4$. We also note that in this range of $\lambda$, the difference scheme $(7)$ is stable.

Next, we define $D^{n}(u, v):=C^{n}(u, v)-u v$; the exten- sion to all $I^{2}$ has been made similarly above. It then follows that $\left\{D_{i, j}^{n}:=C_{i, j}^{n}-u_{i} v_{j}\right\}_{n=0,1,2, \ldots}$ satisfies the system of difference equations (7) with the null boundary conditions. Consequently we see that

$$
\max _{(u, v) \in \boldsymbol{I}^{2}}\left|D^{n}(u, v)\right| \leq K \theta^{n},
$$

for some constants $K, \theta$ with $0<\theta<1$, provided $\lambda<$ $1 / 4$. In particular, we have $D^{n} \rightarrow 0$ as $n \rightarrow \infty$ uniformly on $\boldsymbol{I}^{2}$.

To summarize, we have established the next theorem.

Theorem 3 For any initial copula $C_{0}$, there exists a sequence of copulas $\left\{C^{n}(u, v)\right\}_{n=0,1,2, \ldots}$, which satisfy the system of difference equations (7) at every $\left\{\left(u_{i}, v_{j}\right)\right\}_{i, j=0,1, \ldots, N}$. As $n \rightarrow \infty$, it follows that

$$
C^{n}(u, v) \rightarrow u v \quad \text { uniformly on } I^{2} \text {. }
$$

\section{Measure of dependence}

It is an important subject for research to quantitatively estimate the dependence relation between random variables. For this purpose, several measures of association have been already introduced so far. We recall, as widely known examples, the population version of Kendall's tau and the Spearman's rho, which will be denoted by $\tau$ and $\rho$, respectively.

The formulas for $\tau$ and $\rho$ in terms of copula function is well known. There is also the formulas with respect to the empirical copulas (see Section 5.6 of [6]), which can be utilized for our discretized family of copulas. For the completeness of our exposition, we here reproduce them.

$$
\begin{aligned}
\tau & =\frac{2 N}{N-1} \sum_{i, j=2}^{N}\left(C_{i, j}^{n} C_{i-1, j-1}^{n}-C_{i, j-1}^{n} C_{i-1, j}^{n}\right), \\
\rho & =\frac{12}{N^{2}-1} \sum_{i, j=1}^{N}\left(C_{i, j}^{n}-u_{i} v_{j}\right) .
\end{aligned}
$$

Thanks to these formulas, the convergence as $n \rightarrow \infty$ is deduced directly, which is read as follows.

Theorem 4 For any initial copula $C_{0}$, a sequence of copulas $\left\{C^{n}(u, v)\right\}_{n=0,1,2, \ldots}$ proved in Theorem 3 fulfills

$$
|\tau|,|\rho| \rightarrow 0 \quad \text { exponentially as } n \rightarrow \infty \text {. }
$$

Sketch of Proof In view of (9) and the uniform bound $\max _{(u, v) \in I^{2}}|C(u, v)| \leq 1$, we assert that

$$
\max _{i, j=0,1, \ldots, N}\left|C_{i, j}^{n}-u_{i} v_{j}\right| \rightarrow 0
$$

exponentially as $n \rightarrow \infty$. Taking into account that

$$
u_{i} v_{j} u_{i-1} v_{j-1}-u_{i} v_{j-1} u_{i-1} v_{j}=0,
$$

we see immediately the desired result.

(QED)

\section{Numerical experiments}

The time evolution of copulas in discrete processes have strong affinity to their numeric solutions. Thus we can construct the copulas in accordance with (7) in Theorem 3. For examples, we calculate a Clayton copula $C(u, v)=\left(u^{-\theta}+v^{-\theta}-1\right)^{-1 / \theta}(0<\theta<\infty)$ and its time 
Time evolution of Densities of time evolution of

Clayton copula with $\theta=5 \quad$ Clayton copula with $\theta=5$

$t=0$ (Clayton copula)

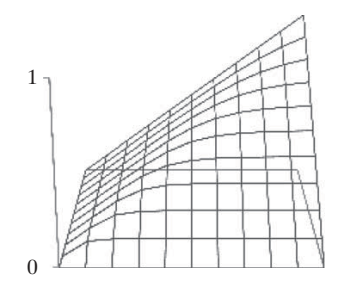

$t=3 / 50$

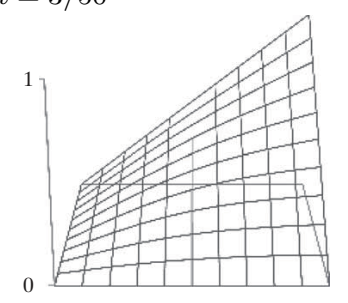

$t=\infty$ (Harmonic copula)

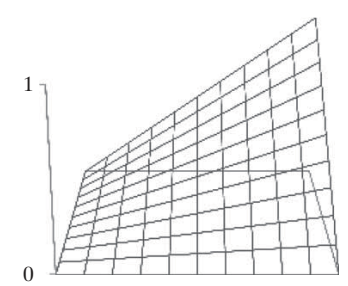

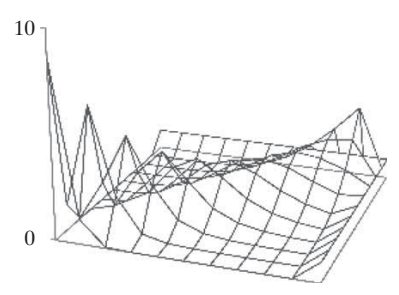
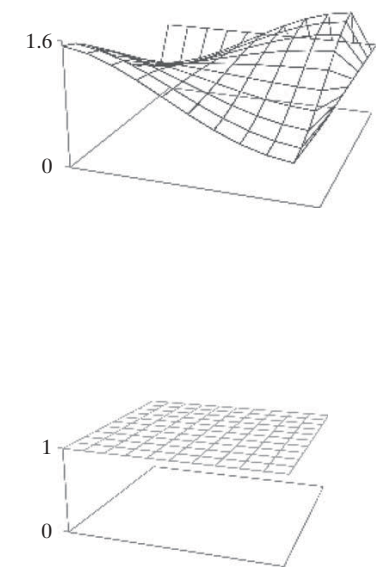

Fig. 1. Time evolution copulas.

evolution in the left side of Fig. 1. As the time evolves, the copulas are smoothed and converge to the harmonic (independence) copula $\Pi$.

We are also able to compute densities of above copulas by the following formula.

The density $=\frac{C_{i, j}^{n}-C_{i, j+1}^{n}-C_{i+1, j}^{n}+C_{i+1, j+1}^{n}}{(\Delta u)(\Delta v)}$.

As an example, we calculate densities of Clayton copula and its time evolution, which are depicted in the right side of Fig. 1. According to the time evolution, the densities of copulas are seen to be smoothed and converge to a flat surface of density one, which is the density of the independence copula $\Pi$.

\section{Discussions}

As stated in Section 1, copulas are employed in quantitative risk management (QRM) for financial engineering, actuarial science, seismology and so on. There are many risk elements and their dependencies are very critical for risk management, especially measuring aggregated risks. Recently copulas are recognized sophisticated method to express quantitatively dependencies between risks. QRM is of use for various purposes. For examples, state dependencies may be enough for solvency regulation purpose, but dynamic dependencies must play important roles in QRM for catastrophic events, such as earthquake, typhoon and financial crisis. We have constructed the time evolution of copulas for the purpose of analyzing dy-

namic dependencies, which are concordant with Brownian motion. Unfortunately they have smoothing nature and our family of time evolving copulas converges to the harmonic copula, which means the independence relation.

Probably in many aspects of applications, the time evolution toward the intended dependence will be much relevant. Thus we propose the backward type of evolution of copulas, which is obtained by transforming the opposite direction of (7):

$$
C^{T}(u, v):=C_{T}(u, v),
$$

where $C_{T}$ denotes given maturity copula and the system of difference equations is

$$
\begin{aligned}
& \frac{C_{i, j}^{T-(n+1) h}-C_{i, j}^{T-n h}}{\Delta t} \\
& =\frac{C_{i+1, j}^{T-n h}-2 C_{i, j}^{T-n h}+C_{i-1, j}^{T-n h}}{(\Delta u)^{2}} \\
& \quad+\frac{C_{i, j+1}^{T-n h}-2 C_{i, j}^{T-n h}+C_{i, j-1}^{T-n h}}{(\Delta v)^{2}} \\
& \quad \text { for } i, j=1,2, \ldots, N-1, \quad n=0,1, \ldots,[T / h],
\end{aligned}
$$

together with the boundary condition (8). We solve (8) and (11) backwardly from the maturity to present.

\section{Acknowledgments}

The authors are grateful to the referee for helpful comments. The second author (N. I.) is supported in part by the grant from the Japan Society for the Promotion of Sciences (No.21540117), as well as the research grant (2011) from the Tokio Marine Kagami Memorial Foundation.

\section{References}

[1] E. W. Frees and E. A. Valdez, Understanding relationships using copulas, N. Amer. Actuarial J., 2 (1998), 1-25.

[2] K. Goda, Statistical modeling of joint probability distribution using copula: Application to peak and permanent displacement seismic demands, Struct. Safety, 32 (2010), 112-123.

[3] K. Goda and G. M. Atkinson, Interperiod dependence of ground-motion prediction equations: A copula perspective, Bull. Seism. Soc. Amer., 99 (2009), 922-927.

[4] R. Lebrun and A. Dutfoy, A generalization of the Nataf transformation to distributions with elliptical copula, Probab. Eng. Mech., 24 (2009), 172-178.

[5] A. J. McNeil, R. Frey and P. Embrechts, Quantitative Risk Management, Princeton Univ. Press, Princeton, 2005.

[6] R. B. Nelsen, An Introduction to Copulas, 2nd edition, Springer Series in Statistics, Springer, New York, 2006.

[7] H. Tsukahara, Copulas and their applications (in Japanese), Jpn J. Appl. Statist., 32 (2003), 77-88.

[8] Y. Yoshizawa, Modeling for the enterprise risk management (in Japanese), Sonpo-Soken Report, 90 (2009), 1-49.

[9] Y. Yoshizawa, Risk management of extremal events (in Japanese), Sonpo-Soken Report, 92 (2010), 35-90.

[10] R. W. J. van den Goorbergh, C. Genest and B. J. M. Werker, Bivariate option pricing using dynamic copula models, Insurance: Math. Econ., 37 (2005), 101-114.

[11] A. Sklar, Random variables, joint distribution functions, and copulas, Kybernetika, 9 (1973), 449-460.

[12] A. J. Patton, Modelling asymmetric exchange rate depen- 
dence, Int. Econ. Rev., 47 (2006), 527-556.

[13] N. Ishimura and Y. Yoshizawa, On time-dependent bivariate copulas, Theor. Appl. Mech. Jpn, 59 (2011), 303-307.

[14] N. Ishimura and Y. Yoshizawa, A note on the time evolution of bivariate copulas, in: Proc. of FAM2011, Sofia Univ., to appear.

[15] Y. Yoshizawa and N. Ishimura, Time Evolution Copulas and Rank Correlation (in Japanese), in: Proc. of JCOSSAR 2011. 